\title{
Pós-estratificação em inventário florestal da vegetação arbórea-arbustiva
}

\author{
Post-stratification in forest inventory of tree-shrub vegetation
}

\author{
Gabriela Paranhos Barbosa1, Gilciano Saraiva Nogueira ${ }^{2}$, Marcio Leles Romarco de Oliveira², \\ Evandro Luiz Mendonca Machado ${ }^{2}$, Renato Vinícius Oliveira Castro ${ }^{3}$ e Gleyce Campos Dutra ${ }^{2}$
}

\begin{abstract}
Resumo
Este trabalho teve como objetivo analisar procedimentos de pós-estratificação em inventário florestal da vegetação arbórea-arbustiva, realizado em uma área de transição entre Floresta Estacional Semidecidual e Cerrado sensu stricto. Foram alocadas sistematicamente 119 parcelas de $20 \times 50 \mathrm{~m}$ e amostrados todos os indivíduos arbóreo-arbustivos. Dois tipos de pós-estratificação das parcelas foram efetuados: pela fitofisionomia e por agrupamento de parcelas distribuídas sistematicamente. Avaliou-se a precisão do inventário florestal, com o uso dos procedimentos de pós-estratificação adotados no estudo. A pós-estratificação pela fitofisionomia gerou os estratos A, que representa a fitofisionomia Floresta Estacional Semidecidual; e $\mathrm{B}$, que representa o Cerrado sensu stricto. A pós-estratificação por agrupamento de parcelas distribuídas sistematicamente definiu três estratos $\left(\mathrm{E}_{1}, \mathrm{E}_{2}\right.$ e $\left.\mathrm{E}_{3}\right)$ e, pelas estatísticas do inventário, essa apresentou-se mais precisa do que a pós-estratificação pela fitofisionomia, com o menor erro de amostragem $(3,72 \%)$ e menor amplitude do intervalo de confiança.
\end{abstract}

Palavras-chave: Amostragem sistemática, análise de agrupamento, transição.

\begin{abstract}
This study aimed to analyze post-stratification procedures for forest inventory of the tree-shrub vegetation, effectuated in a transition area between Semi-deciduous Tropical Forest and Cerrado sensu stricto. One hundred and nineteen plots of $20 \times 50 \mathrm{~m}$ have been systematically allocated, with all arboreal-shrubby individuals sampled. Two types of plots were used: post-stratification by phyto-physiognomy and by grouping systematically distributed plots. The accuracy of the forest inventory was evaluated through the application of post-stratification procedures used in the study. The post-stratification by phyto-physiognomy generated strata A, representing the Semi-deciduous Tropical Forest; and B, the Cerrado sensu stricto. The post-stratification by grouping plots, systematically distributed defined three strata $\left(E_{1}, E_{2}\right.$ and $\left.E_{3}\right)$. By the statistics obtained from the inventory, this was more accurate than the post-stratification by phyto-physiognomy, with the smallest sampling error (3.72\%), and lower amplitude of the confidence interval.
\end{abstract}

Keywords: Systematic sampling, cluster analysis, transition.

\section{INTRODUÇÃO}

Inventário florestal é um procedimento para a obtenção de informações sobre a quantidade, a qualidade e a condição dos recursos florestais, da vegetação e dos componentes associados bem como das características das áreas onde a floresta está localizada (HUSCH et al., 2003).

Procedimentos de inventário florestal, definidos em função dos seus objetivos, dos recursos disponíveis e da precisão requerida, apresentam-se como a forma mais eficiente de se conhecer uma floresta e inferir sobre suas características.

O tipo de inventário mais realizado no mundo é o inventário por amostragem. Porém, só se obtém sucesso por este tipo de inventário se o procedimento de amostragem utilizado for capaz de fornecer estimativas reais das características de interesse da população, tendo como base apenas uma amostra da mesma (THOMPSON; SEBER, 1996; SOUZA et al., 2006). Na busca por

${ }^{1}$ Doutorando no Departamento de Engenharia Florestal. UFVJM - Universidade Federal dos Vales do Jequitinhonha e Mucuri - Campus JK - Rodovia MGT 367 KM 583, n. 5000 - Alto Jacuba, Diamantina, MG, Brasil. E-mail: barbosagabriella@hotmail.com.

2Professor Adjunto no Departamento de Engenharia Florestal. UFVJM - Universidade Federal dos Vales do Jequitinhonha e Mucuri - Campus JK - Rodovia MGT 367 KM 583, n. 5000 - Alto Jacuba, Diamantina, MG, Brasil. E-mail: nogueirags@gmail.com; marcioromarco@gmail.com; machadoelm@gmail.com; gleycedutra@yahoo.com.br.

3Professor Adjunto no Departamento de Ciências Agrárias. UFS - Universidade Federal de São João Del-Rei CSL - Campus Sete Lagoas, Rua Sétimo Moreira Martins - Itapoã II - 35702031 - Sete Lagoas, MG, Brasil. E-mail: castrorvo@ymail.com. 
este sucesso, é de grande importância a definição dos procedimentos e técnicas mais adequadas à determinada área.

A pós-estratificação é uma técnica de amostragem utilizada em inventários florestais da vegetação, que consiste na divisão da amostra em estratos após a coleta de dados (LUND; THOMAS, 1989), ou seja, realiza-se a coleta dos dados, identifica-se a variabilidade da população e, a partir desse conhecimento, efetua-se a estratificação. Os estratos são formados agrupando-se parcelas com características semelhantes, a fim de se obter maior precisão das estimativas no inventário florestal.

No caso de florestas nativas, nas quais as informações da área são geralmente desconhecidas antes do levantamento de campo, o uso da pós-estratificação se torna uma alternativa. Porém, como a população nesses casos é muito variável em espécies, idades das árvores e condições de locais, a estratificação torna-se mais complexa, pois são muitas características variando ao mesmo tempo e todas devem ser consideradas (SOUZA, 1989; HUSCH et al., 2003).

Os procedimentos de estratificação mais comuns utilizados em florestas nativas são aqueles que utilizam imagens e fotografias aéreas (interpretação visual) (LUND; THOMAS, 1989), e informações qualitativas da floresta, como suas análises da composição florística e estruturas fitossociológicas, para constituir os estratos. Outro procedimento que tem conquistado espaço é o que utiliza informações quantitativas, como volume e área basal, para definição dos estratos. Para florestas nativas, o uso de técnicas de análise multivariada (SOUZA, 1989) pode ser uma alternativa para facilitar a definição dos estratos em situações onde diversas características variam ao mesmo tempo.

Conhecer a área é o passo fundamental para o sucesso de uma estratificação, pois, definir bem os estratos, para que eles sejam os mais homogêneos possíveis internamente e distintos entre si, permite que a variabilidade da área seja amenizada e garante a obtenção de estimativas precisas da população (LOETSCH; HALLER, 1964). A pós-estratificação possibilita este efetivo conhecimento das informações da área, pelo fato de os dados serem conhecidos antes de uma estratificação.

Nesse sentido o objetivo do trabalho é analisar procedimentos de pós-estratificação em inventário florestal de vegetação arbórea-arbustiva, realizado em uma área de transição entre Floresta Estacional Semidecidual e Cerrado sensu stricto.

\section{MATERIAL E MÉTODOS}

\section{Caracterização da área e coleta de dados}

O estudo foi realizado em uma formação florestal, pertencente à Fazenda Cipó, com área total de 1.696,79 ha, localizada no município de Francisco Dumont, na região centro-norte do estado de Minas Gerais, nas coordenadas geográficas $17^{\circ} 13^{\prime} 26^{\prime \prime} \mathrm{S}$ e $44^{\circ} 08^{\prime} 57^{\prime \prime} \mathrm{O}$. A altitude da região varia de 559 a 1.391 metros sobre o nível do mar. O clima é classificado como semiárido quente, do tipo BSh, de acordo com a classificação de Köppen, com temperaturas médias em torno de $22,4^{\circ} \mathrm{C}$, e índice pluviométrico próximo a 1.082,3 mm por ano (IBGE, 2001). A vegetação nativa predominante da região é o Cerrado sensu stricto, porém apresenta muitas áreas de transição com remanescentes de Mata Atlântica (IBGE, 2004), com predomínio da fisionomia Floresta Estacional Semidecidual. Em algumas áreas é possível encontrar formações abertas de Campo Cerrado.

Foram utilizados dados dendrométricos e florísticos obtidos por meio de inventário florestal, em que se excluíram as áreas de formações abertas da amostragem da vegetação arbórea-arbustiva, e no restante da área distribuíram-se 119 parcelas de $20 \times 50 \mathrm{~m}\left(1.000 \mathrm{~m}^{2}\right)$ de maneira sistemática, totalizando um universo amostral de 11,90 ha.

Em cada unidade amostral foram mensurados os indivíduos arbóreos-arbustivos vivos com diâmetro a $1,30 \mathrm{~m}(D A P)=5 \mathrm{~cm}$. Todos os indivíduos foram contabilizados, identificados, e sua circunferência a 1,30 m (CAP) foi mensurada com o auxílio de uma fita métrica e a altura total (Ht) foi estimada por comparação com uma vara graduada. Os dados foram coletados entre os meses de maio e junho de 2012.

A identificação das espécies foi realizada posteriormente em laboratório, com a observação de material botânico coletado pelas equipes de campo. As identificações seguiram consultas à literatura, tais como o sistema APG III (APGIII, 2009).

O volume total da parcela foi obtido por meio do somatório dos volumes individuais de cada árvore. Para estimação do volume das árvores foram empregadas equações volumétricas conforme 
CETEC (1995), sendo a equação de mata secundária (1) utilizada para estimar o volume nas parcelas com predomínio da Floresta Estacional Semidecidual e a equação de Cerrado (2) para estimar o volume nas parcelas com predomínio de formações savânicas.

$$
\begin{aligned}
& \text { Mata secundária: } V t=0,000074 * d a p^{1,707348 * H t^{1,16873}}, R^{2}=97,3 \% \\
& \text { Cerrado: } V t=0,000065661 * d a p^{2,475293} * H t^{0,300022}, R^{2}=98,1 \%
\end{aligned}
$$

em que: $V t=$ volume total com casca em m${ }^{3} ; D A P=$ diâmetro a 1,30 m, em cm; Ht = altura total, em $\mathrm{m}$; e $R^{2}=$ coeficiente de determinação.

\section{Pós-estratificação e estatísticas do Inventário Florestal}

Foram considerados dois procedimentos de pós-estratificação para definição dos estratos no estudo. O primeiro procedimento, denominado como pós-estratificação pela fitofisionomia, utilizou a interpretação visual de imagens aéreas, associada à interpretação de análises florísticas e de estruturas fitossociológicas da formação florestal para a definição dos estratos. Inicialmente, utilizou-se o método da interpretação visual que se baseia nos princípios de fotointerpretação, em que a segmentação é determinada por um grau de homogeneidade, que permite um agrupamento de pixels em função da tonalidade (espectral) e da forma (suavização e compactação) do objeto da imagem (ANTUNES, 2003). Posteriormente, a composição florística e as estruturas fitossociológicas foram utilizadas para confirmar a divisão realizada pela imagem e nomear as fitofisionomias. Cada uma dessas fitofisionomias representou um estrato na pós-estratificação. A área dos estratos neste procedimento foi obtida por meio da análise das imagens aéreas em um ambiente SIG (Sistema de Informações Geográficas).

O segundo procedimento utilizou técnicas de análise multivariada para agrupar parcelas com características semelhantes em estratos. Isso só foi possível devido à maneira sistemática que as parcelas foram distribuídas na área. Na amostragem sistemática, as parcelas ficam distribuídas de forma uniforme e ocupam toda a área da população, o que garante que todas sejam equivalentes e possuam a mesma probabilidade de serem selecionadas em um esquema de agrupamento. Mesmo que essas parcelas não estejam próximas espacialmente, a sistematização permite que elas sejam agrupadas para formação de estratos. Esse procedimento foi aqui denominado pós-estratificação por agrupamento de parcelas distribuídas sistematicamente.

A pós-estratificação por agrupamento de parcelas distribuídas sistematicamente consistiu inicialmente na quantificação da área basal e do volume para cada unidade de amostra lançada na área. De posse dessas informações, procedeu-se à elaboração de uma matriz $X$ de dados, em que cada variável $x_{i j}$ representa o $i$-ésimo volume e área basal na j-ésima parcela. Assim, realizou-se a pós-estratificação das parcelas, utilizando a matriz $X$ como entrada de dados para as análises de agrupamento e discriminante. Como critério de agrupamento, foram utilizadas a distância euclidiana e o método de Ward (SOUZA; SOUZA, 2006). Essas análises foram processadas utilizando o software Statistica 10.0 (STATSOFT, 2010).

Para a definição do número de estratos mais homogêneos, e das parcelas que o compunham, traçou-se a linha de corte ou linha fenon, que é uma linha paralela ao eixo horizontal do dendrograma obtido da análise de agrupamento (HOSOKAWA et al., 1990).

Por fim, aplicou-se a análise discriminante para verificar a veracidade da distinção e classificação das parcelas em estratos homogêneos.

O cálculo da área dos estratos formados se deu pela proporção do número de parcelas agrupadas em cada estrato, em relação à área total. Isto só foi possível devido ao esquema sistemático de distribuição das parcelas, em que todas possuem mesma probabilidade de seleção.

Procedeu-se também uma simulação em que o número de parcelas da amostragem foi reduzido, em cerca de 50\%, seguindo um esquema em que se retirou as parcelas de linhas intercaladas. Esta simulação objetivou avaliar o efeito da redução da intensidade amostral na precisão do inventário florestal, com o uso dos procedimentos de pós-estratificação adotados no estudo. A redução manteve o esquema de sistematização, preservando o intervalo entre as unidades de amostra e garantindo uma amostragem de forma a ocupar inteiramente a área da população. Após a redução, novas estratificações foram realizadas por ambos os procedimentos de pós-estratificação, entretanto com menor número de unidades de amostra.

Sci. For., Piracicaba, v. 45, n. 115, p. 445-453, set. 2017 DOI: dx.doi.org/10.18671/scifor.v45n115.03 
As estatísticas do inventário florestal foram obtidas por estimadores de diferentes processos de amostragem que foram posteriormente comparados. Procedeu-se as análises utilizando-se dos estimadores da amostragem casual simples (ACS), considerando a área sem estratificação, de acordo com Husch et al. (2003). Realizou-se ainda o processamento do inventário florestal utilizando as diferenças sucessivas, no qual o cálculo do erro-padrão da média foi obtido pelo estimador descrito por Loetsch e Haller (1964). Nessa situação também foi considerada a área sem estratificação. Por fim, as estatísticas do inventário florestal foram obtidas por meio do processo de amostragem estratificada (AE), considerando os dois procedimentos de pós-estratificação: a pós-estratificação pela fitofisionomia; e a pós-estratificação por agrupamento de parcelas distribuídas sistematicamente. Os estimadores populacionais da amostragem estratificada também foram calculados de acordo com Husch et al. (2003).

Realizou-se, ainda, o processamento das estatísticas do inventário, considerando a redução da intensidade amostral, em cerca de 50\%. Essas estimativas da população, baseadas na amostra de tamanho menor, foram obtidas por meio de todos os processos de amostragem e procedimentos de pós-estratificação empregados no estudo.

Ao final das análises, os erros de amostragem e demais estatísticas dos inventários florestais foram comparados, a fim de se avaliar a precisão das estimativas e a eficiência no uso dos procedimentos de pós-estratificação.

\section{RESULTADOS E DISCUSSÃO}

As análises da composição florística, da estrutura fitossociológica e da estrutura paramétrica, juntamente com análise de imagens aéreas, permitiram caracterizar a área de estudo como de transição, com divisão bem definida entre as fitofisionomias Floresta Estacional Semidecidual (113 espécies; 1.147,73 árvores/ha; e 13,29 m²/ha de área basal) e Cerrado sensu stricto (121 espécies; 1.767,95 árvores/ha; e 10,23 $\mathrm{m}^{2} /$ ha de área basal), ambas com diversidade elevada e em estágio inicial de sucessão. Assim, por meio da pós-estratificação pela fitofisionomia formou-se dois estratos na área: o estrato A que representa a fitofisionomia Floresta Estacional Semidecidual com 756,9395 ha e o estrato B que representa a fitofisionomia Cerrado sensu stricto com 384,0468 ha de área (Figura 1).

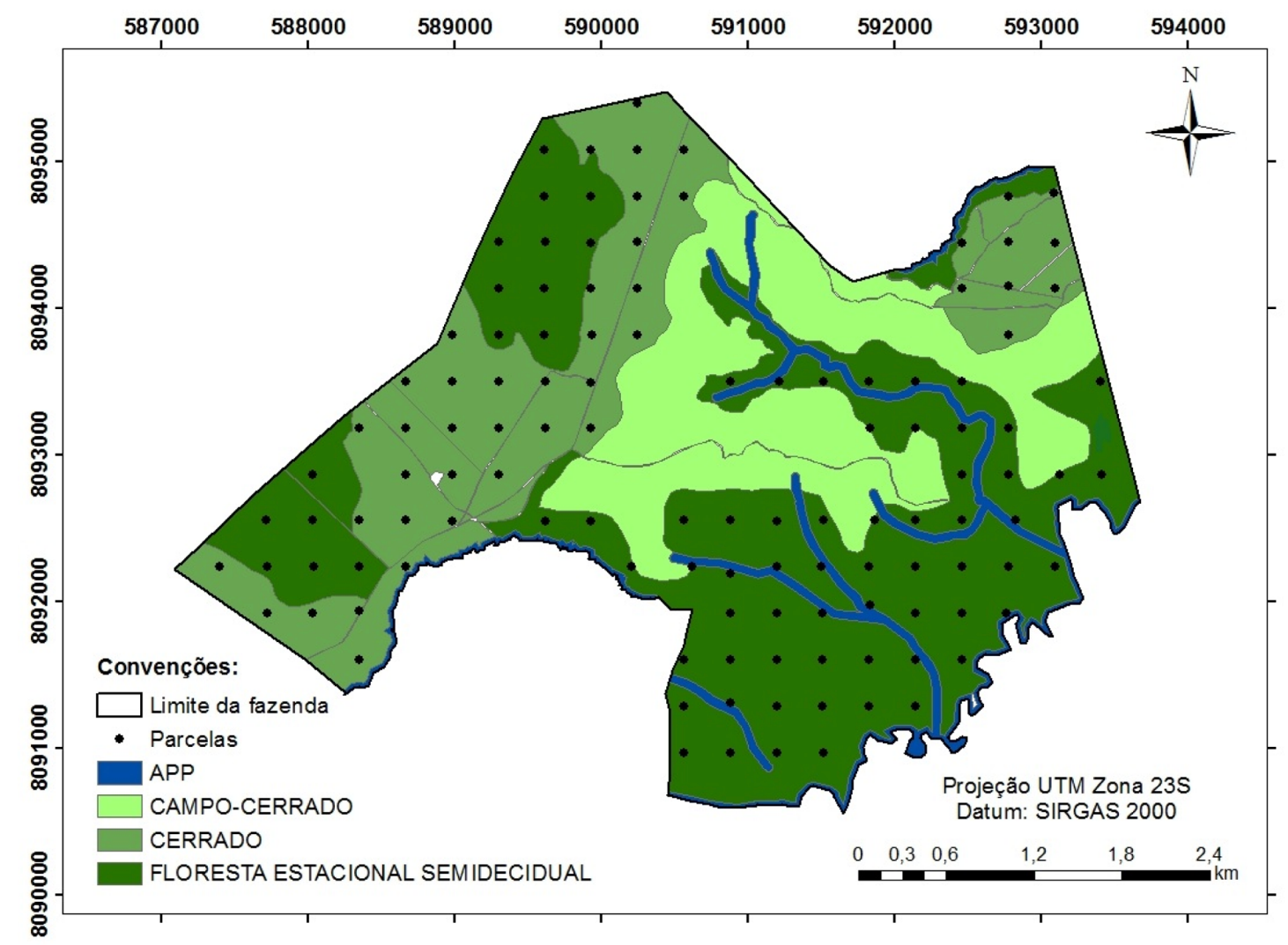

Figura 1. Fitofisionomias presentes na formação florestal em estudo.

Figure 1. Fitofisionomy present in the forest formation studied. 
Já por meio da pós-estratificação por agrupamento de parcelas distribuídas sistematicamente, na análise do dendrograma, traçada no nível de homogeneidade de 4\%, três estratos ficaram definidos na área do estudo, sendo: estrato $\mathrm{E}_{1^{\prime}}$ contendo 33 parcelas e uma área de 316,41 ha; estrato $\mathrm{E}_{2^{\prime}}$ contendo 39 parcelas e uma área de 373,94 ha; e estrato $\mathrm{E}_{3^{\prime}}$ contendo 47 parcelas e uma área de 450,65 ha (Figura 2).

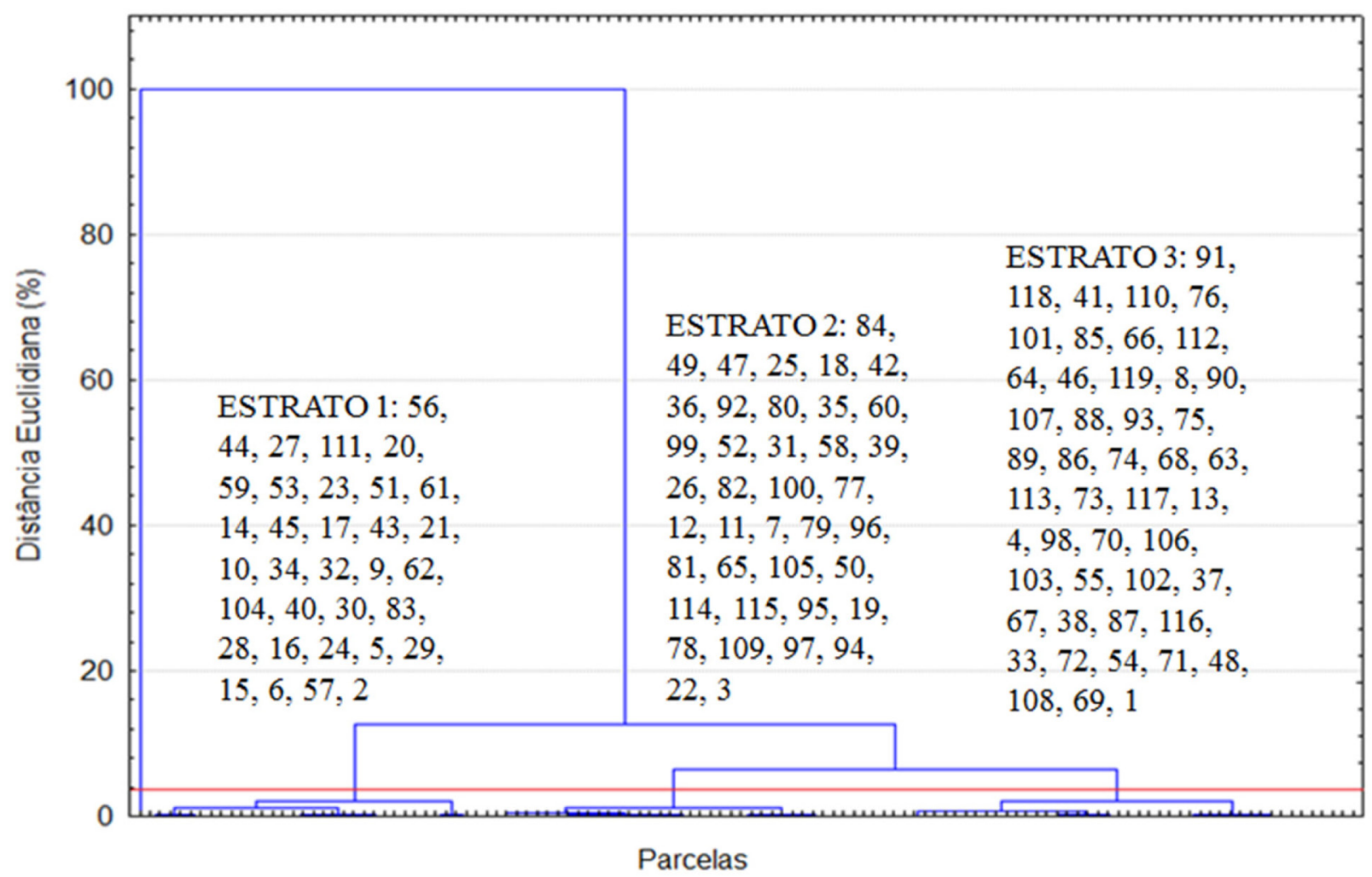

Figura 2. Dendrograma gerado a partir da análise agrupamento, para as 119 parcelas lançadas na formação florestal em estudo.

Figure 2. Dendrogram generated from the cluster analysis, for 119 plots allocated in the forest formation studied.

O dendrograma obtido da análise de agrupamento apresenta nas ordenadas a dissimilaridade, em porcentagem, variando de zero (máxima similaridade) a 100 (máxima dissimilaridade), e nas abscissas, as parcelas agrupadas.

A análise discriminante, processada para verificar a veracidade da distinção e classificação das parcelas em estratos homogêneos, apresentou probabilidades de 93,94\%; 100\%; e 97,87\%, respectivamente, nos estratos $E_{1}, E_{2} e_{3}$. O procedimento final de classificação indicou que $97,48 \%$ das unidades de amostra foram corretamente classificadas nos estratos.

Segundo Souza et al. (2014) a análise discriminante é uma técnica útil não só para avaliar a consistência da análise de agrupamento, mas também é utilizada para alocar dados aos grupos com maior afinidade estatística. Nesse caso, a análise discriminante mostra que as unidades de amostra apresentaram características volumétricas e de área basal semelhantes dentro do estrato ao qual pertencem, e heterogeneidade entre os estratos.

Considerando a simulação em que se reduziu 50\% do número de parcelas amostradas, chegou-se a um número de 58 parcelas distribuídas de forma sistemática na área. No novo dendrograma obtido da pós-estratificação por agrupamento de parcelas distribuídas sistematicamente, considerando menor número de parcelas (Figura 3), foi traçada uma linha de corte no nível de homogeneidade em $20 \%$, destacando-se os 3 estratos formados $\left(E_{1}, E_{2^{\prime}}, E_{3}\right)$, sendo: estrato $E_{1}$ contendo 19 parcelas e área de 373,77 ha, estrato $\mathrm{E}_{2}$ contendo 25 parcelas e área de 491,81 ha e estrato $\mathrm{E}_{3}$ contendo 14 parcelas e área de 275,41 ha. 


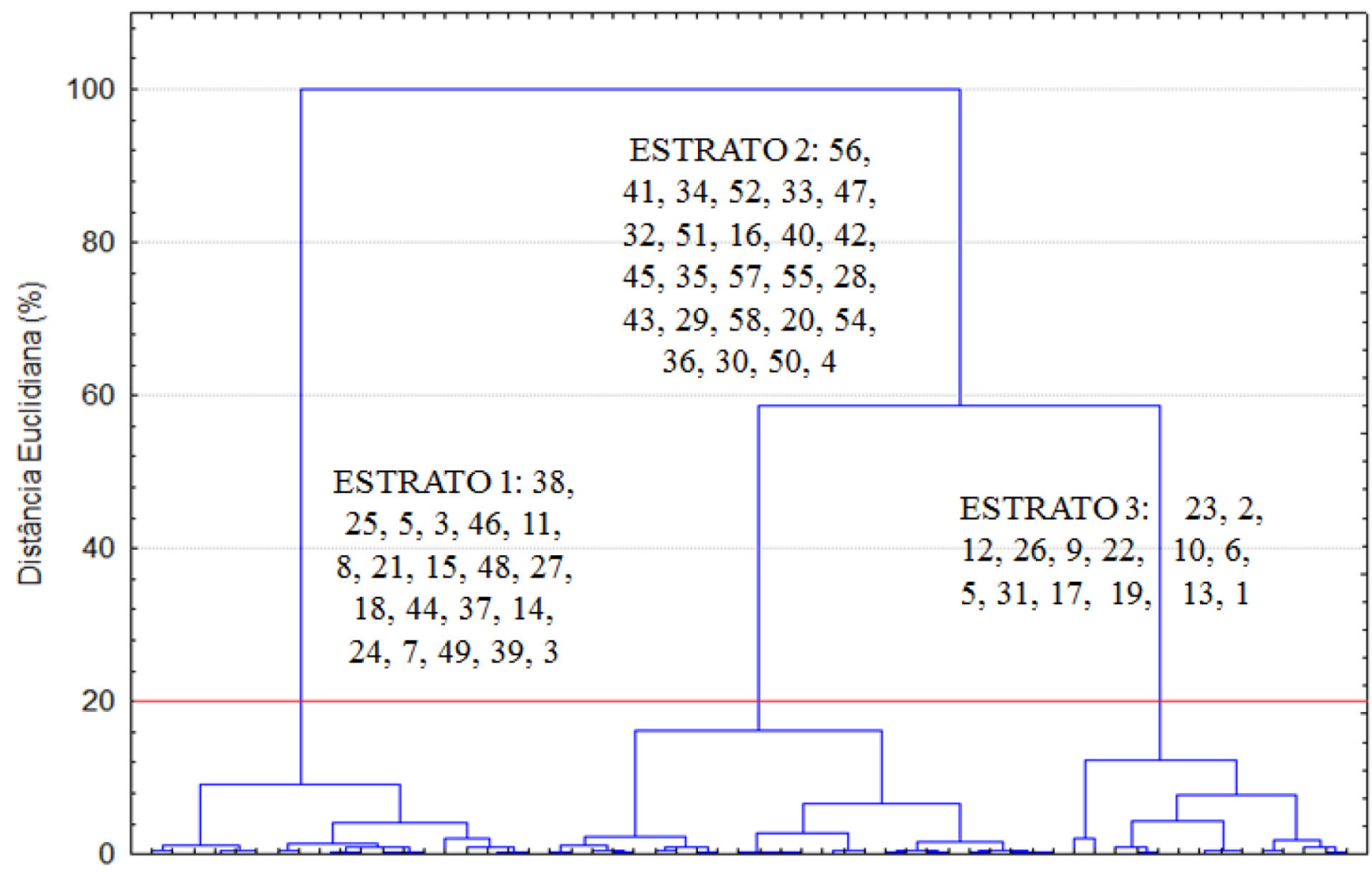

Parcelas

Figura 3. Dendrograma gerado a partir da análise agrupamento, considerando a redução para 58 parcelas na formação florestal em estudo.

Figure 3. Dendrogram generated from the cluster analysis, considering the reduction to 58 plots in the forest formation studied.

A análise discriminante processada para verificar a veracidade da distinção e classificação das parcelas em estratos homogêneos após a simulação da redução do número de parcelas garantiu um acerto de $96,55 \%$.

Os resultados dos estimadores do inventário florestal empregando-se as fórmulas dos processos de amostragem casual simples e das diferenças sucessivas sem considerar a pós-estratificação, e da amostragem estratificada considerando a pós-estratificação pela fitofisionomia e pelo agrupamento de parcelas distribuídas sistematicamente estão apresentados na Tabela 1.

Tabela 1. Análise estatística das estimativas por diferentes processos de amostragem e pós-estratificação para as 119 parcelas lançadas no inventário florestal realizado na formação florestal em estudo.

Table 1. Statistical analysis of estimates by different sampling processes and post-stratification for 119 plots allocated in the forest formation studied.

\begin{tabular}{lcccc}
\hline Estimadores & $\begin{array}{c}\text { Casual } \\
\text { Simples }\end{array}$ & $\begin{array}{c}\text { Diferenças } \\
\text { Sucessivas }\end{array}$ & $\begin{array}{c}\text { Estratificada pela } \\
\text { fitofisionomia }\end{array}$ & $\begin{array}{c}\text { Estratificada pela } \\
\text { Análise de Agrupamento }\end{array}$ \\
\hline Volume médio $(\bar{y})$ & 5,47 & 5,47 & 5,53 & 5,47 \\
Variância $\left(\delta^{2}\right)$ & 8,83 & 8,83 & 0,07 & 0,01 \\
Erro padrão da média $\left(S_{\bar{y}}\right)$ & 0,27 & 0,23 & 0,26 & 0,10 \\
Erro de Amostragem $(\%)$ & 9,86 & 8,49 & 9,18 & 3,72 \\
Coeficiente de Variação $(\%)$ & 54,34 & 54,34 & 50,88 & 20,61 \\
Estimativa de Produção $\left(\mathrm{m}^{3}\right)$ & 62403,80 & 62403,80 & 63077,92 & 62403,80 \\
Intervalo de confiança $\left(\mathrm{m}^{3}\right)$ & $\pm 6155,57$ & $\pm 5295,68$ & $\pm 5793,55$ & $\pm 2321,7$ \\
\hline
\end{tabular}

A pós-estratificação por agrupamento de parcelas distribuídas sistematicamente apresentou-se o mais preciso de todos os processos, com o menor erro de amostragem (3,72\%), e menor amplitude do intervalo de confiança.

A Amostragem Casual Simples (ACS) apresentou o maior erro de todos os processos $(9,86 \%)$ e o maior intervalo de confiança $\left(62403,80 \pm 6155,57 \mathrm{~m}^{3}\right)$. Como a área do estudo é uma área de transição, sua população apresenta grande variabilidade em suas características, e o processo de ACS apresenta menor capacidade de amenizar esta variabilidade. 
A amostragem por diferenças sucessivas apresentou-se como o segundo melhor processo em relação ao erro de amostragem $(8,49 \%)$. Porém, por se tratar de uma área de transição, não é certa a existência de menores diferenças entre as unidades de amostra próximas do que entre aquelas mais distantes, já que parcelas próximas podem pertencer à fitofisionomias diferentes.

A pós-estratificação pela fitofisionomia apresentou redução da variância em relação a ACS e as diferenças sucessivas. Porém, não se apresentou tão precisa quanto ao erro de amostragem ao ser comparada com a pós-estratificação por agrupamento de parcelas distribuídas sistematicamente. Esse fato pode ser atribuído às imagens aéreas da área do estudo, que não permitiram identificar áreas com maior ou menor heterogeneidade, ou ainda devido às dimensões da área que não foram bem representadas na imagem, impossibilitando que boas classificações fossem realizadas a partir daquela imagem.

A pós-estratificação pela fitofisionomia é um dos procedimentos mais empregados na área florestal, e o fato da fotointerpretação muitas vezes não conseguir revelar áreas com maior ou menor heterogeneidade, induz à manipulação imprópria dos dados pelo inventariante, a fim de que os inventários tenham as medidas de precisão dentro do estabelecido pelos órgãos ambientais. Dessa forma, a pós-estratificação por agrupamento de parcelas distribuídas sistematicamente mostra-se um procedimento muito adequado, pois, por ser altamente preciso, intervenções dessa natureza não são necessárias.

É importante ressaltar que, para fins de plano de corte, os valores dos erros de amostragem obtidos empregando-se as fórmulas da amostragem casual simples, da diferença sucessiva e da amostragem estratificada foram satisfatórios (menor que 10\%). Assim, o tamanho da amostra foi suficiente para gerar um erro de amostragem inferior a um erro pré-estabelecido de $10 \%$, independentemente do processo empregado.

Ao reduzir o tamanho da amostra para 58 parcelas (Tabela 2) verifica-se que os erros de amostragem obtidos pelos estimadores da amostragem casual simples, das diferenças sucessivas e da amostragem estratificada, considerando a pós-estratificação pela fitofisionomia, foram superiores a 10\%.

Tabela 2. Análise estatística das estimativas por diferentes processos de amostragem e pós-estratificação considerando a redução para 58 parcelas amostradas no inventário florestal realizado na área do estudo.

Table 2. Statistical analysis of estimates by different sampling processes and post-stratification considering the reduction to 58 plots in the forest inventory conducted in the study area.

\begin{tabular}{lcccc}
\hline Estimadores & ACS & $\begin{array}{c}\text { Diferenças } \\
\text { Sucessivas }\end{array}$ & $\begin{array}{c}\text { AE pela } \\
\text { fitofisionomia }\end{array}$ & $\begin{array}{c}\text { AE pela Análise de } \\
\text { Agrupamento }\end{array}$ \\
\hline Volume médio $(\bar{y})$ & 5,16 & 5,16 & 5,25 & 5,16 \\
Variância $\left(\delta^{2}\right)$ & 6,93 & 6,93 & 0,11 & 0,02 \\
Erro padrão da média $\left(S_{\bar{y}}\right)$ & 0,35 & 0,33 & 0,33 & 0,13 \\
Erro de Amostragem $(\%)$ & 13,43 & 12,81 & 12,63 & 4,90 \\
Coeficiente de variação $(\%)$ & 51,07 & 51,07 & 48,16 & 18,68 \\
Estimativa de Produção $\left(\mathrm{m}^{3}\right)$ & 58830,60 & 58830,6 & 59863,96 & 58830,6 \\
Intervalo de confiança $\left(\mathrm{m}^{3}\right)$ & $\pm 7899,83$ & $\pm 7533,57$ & $\pm 7559,74$ & $\pm 2881,21$ \\
\hline
\end{tabular}

Percebe-se uma elevação do erro de amostragem e, consequentemente, dos intervalos de confiança, para todos os processos após a redução do tamanho da amostra. Porém, é possível observar que, quando retirada algumas parcelas para análise, mantendo-se o esquema de sistematização na distribuição amostral, a precisão foi ainda muito satisfatória para a pós-estratificação por agrupamento de parcelas distribuídas sistematicamente $(4,9 \%)$. Dessa forma, fica claro que, para a realização de inventários florestais utilizando a pós-estratificação por agrupamento de parcelas distribuídas sistematicamente, o tamanho da amostra necessária para atingir certa precisão é bem menor, o que beneficia muito os trabalhos de coleta de dados, reduzindo os custos do inventário, já que a coleta de dados é a etapa mais onerosa em um inventário florestal (CAMPOS; LEITE, 2013).

Para a pós-estratificação pela fitofisionomia, é necessária uma maior intensidade amostral para que as estimativas sejam precisas, pois apenas um maior tamanho da amostra viabiliza a coleta de dados mais heterogêneos, captando, consequentemente, melhor a variabilidade dos estimadores na área.

Alvarenga et al. (2012), avaliando a redução da intensidade amostral na precisão de um inventário de Cerrado sensu stricto por meio da interpretação visual, encontraram resultados precisos, o que representa redução de tempo e custo para a realização do inventário. No entanto, os autores ressal- 
taram a importância da boa interpretação visual para a obtenção de estratos mais homogêneos que a população como um todo.

Na Figura 4 é possível observar os limites do intervalo de confiança para todos os processos utilizados no estudo em ambos os tamanhos de amostra considerados.

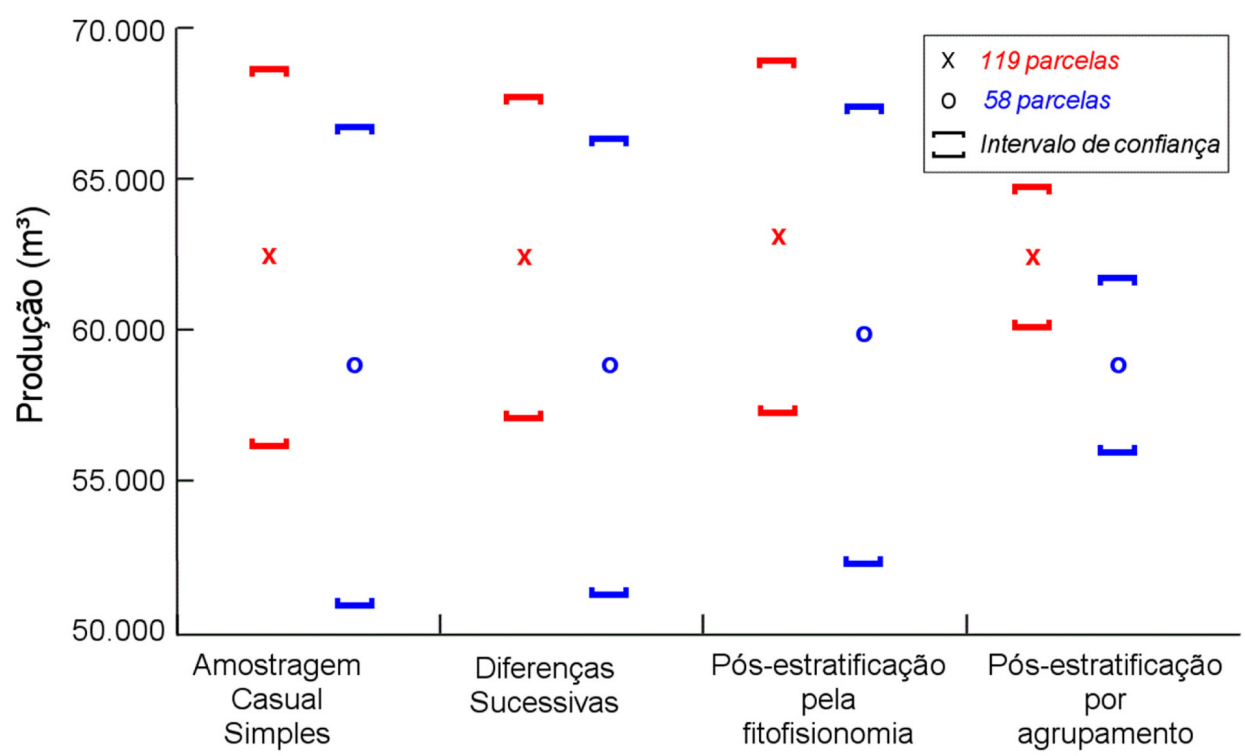

Figura 4. Intervalos de confiança da produção total para a área do estudo, considerando todos os processos de amostragem adotados no estudo para os dois tamanhos da amostra (119 e 58 parcelas).

Figure 4. Confidence intervals of the total production for the study area, considering all sampling processes used in the study for the two sample sizes (119 and 58 plots).

Nota-se que, para o procedimento de pós-estratificação por agrupamento de parcelas distribuídas sistematicamente, independentemente do tamanho da amostra, os intervalos foram consideravelmente inferiores aos demais processos, mostrando-se uma alternativa muito eficiente para a obtenção de estimativas precisas. Os resultados demonstram que esse processo pode ser indicado para florestas naturais, quando não existam mapas ou fotografias disponíveis ou quando a fotointerpretação revelar pouca base para a estratificação, como em pequenas propriedades.

\section{CONCLUSÕES}

O procedimento de pós-estratificação por análise de agrupamento de parcelas distribuídas sistematicamente foi mais eficiente e mais preciso do que o procedimento de pós-estratificação definido com base na fitofisionomia.

O intervalo de confiança obtido pelo procedimento de pós-estratificação pela fitofisionomia não diferenciou consideravelmente dos intervalos obtidos ao considerar a área sem estratificação e empregando-se as fórmulas da amostragem casual simples e das diferenças sucessivas.

A redução do tamanho da amostra não influenciou, ao ponto de ultrapassar os limites pré-estabelecidos pelos órgãos ambientais, a precisão do inventário, obtida pelo procedimento de pós-estratificação por análise de agrupamento de parcelas distribuídas sistematicamente, evidenciando que esse procedimento é capaz de gerar estimativas precisas mesmo com baixa intensidade amostral.

\section{REFERÊNCIAS BIBLIOGRÁFICAS}

ALVARENGA, L. H. V.; MELLO, J.M.; GUEDES, I. C. L.; SCOLFORO, J.R.S. Desempenho da estratificação em um fragmento de Cerrado stricto sensu utilizando interpolador geoestatístico. Revista Cerne, Lavras, v. 18, n. 4, p. 675-681, 2012.

ANTUNES, A. F. B. Classificação de ambiente ciliar baseada em orientação a objeto de imagens de alta resolução espacial. 2003. 147 p. Tese (Doutorado em Ciências Geodésicas) - Universidade Federal do Paraná, Curitiba, 2003. 
APGIII - ANGIOSPERM PHYLOGENY GROUP III. APGIII: An update of the Angiosperm Phylogeny Group classification for orders and families of flowering plants. Botanic Journal Linnean Society, New York, v. 161, n. 2, p. 105-121, 2009.

CAMPOS, J. C. C.; LEITE, H. G. Mensuração Florestal: perguntas e respostas. 4. ed. Viçosa: Editora UFV; 2013. $605 \mathrm{p}$.

CETEC - CENTRO TECNOLÓGICO DE MINAS GERAIS. Determinação de equações volumétricas aplicáveis ao manejo sustentado de florestas nativas do estado de Minas Gerais e outras regiões do país. Belo Horizonte: Fundação Centro Tecnológico de Minas Gerais, 1995. 295 p.

HOSOKAWA, R. T.; SOUZA, A. L.; KIRCHNER, F. F.; MACHADO, S. E. A. Análises multivariadas para manejo de floresta natural na Reserva Florestal de Linhares, Espírito Santo: análises de agrupamento e discriminante. Revista Árvore, Viçosa, v. 14, n. 2, p. 85-101, 1990.

HUSCH, B; BEERS, T. W; KERSHAW JÚNIOR, J. A. Forest Mensuration. 4 ed. New Jersey: John Wiley and Sons LTD., 2003.

IBGE - INSTITUTO BRASILEIRO DE GEOGRAFIA E ESTATÍSTICA. IBGE: Mapa de Vegetação do Brasil. Rio de Janeiro: IBGE, 2004. Escala 1:5.000.000. Disponível a partir de: < ftp://geoftp.ibge.gov.br/mapas/tematicos/ mapas murais/vegetacao.pdf $>$. Acesso em: jun. 2016

IBGE - INSTITUTO BRASILEIRO DE GEOGRAFIA E ESTATÍSTICA. Mapa de Solos do Brasil. Rio de Janeiro: IBGE, 2001. Escala 1:5.000.000. Disponível em: < http://www.dpi.inpe.br/Ambdata/mapa solos.php >. Acesso em: jun. 2016

LOETSCH, F; HALLER, K. A. Forest inventory. Munique: Blv Verlagsgesellschaft, 1964. v. 1, 436 p.

LUND, H. G; THOMAS, C. E. A primer on stand and forest inventory designs. Washington: USDA Forest service, 1989. 96 p. (General Technical Report WO-54).

SOUZA, A. L.; MEDEIROS, R. M.; MATOS, L. M. S.; SILVA, K. R.; CORRÊA, P. A.; FARIAS, F. N. Estratificação volumétrica por classes de estoque em uma floresta ombrófila densa, no município de Almeirim, estado do Pará, Brasil. Revista Árvore, Viçosa, v. 38, n. 3, p. 533-541, 2014.

SOUZA, A. L; SOUZA, D. R. Análise multivariada para estratificação volumétrica de uma floresta ombrófila densa de terra firme, Amazônia Oriental. Revista Árvore, Viçosa, v. 30 n. 1, p. 49-54, 2006.

SOUZA, D. R.; SOUZA, A. L.; LEITE, H. G.; YARED, J. A. G. Análise estrutural em floresta ombrófila densa de terra firme não explorada, Amazônia oriental. Revista Árvore, Viçosa, v. 30, n. 1, p. 75-87, 2006.

SOUZA, A. L. Análise multivariada para manejo de florestas naturais: alternativas de produção sustentada de madeiras para serraria. 1989. 255 p. Tese (Doutorado em Ciência Florestal) - Universidade Federal do Paraná, Curitiba, 1989.

STATSOFT, Inc., Statistica (Data Analysis Software System): version 10.0. 2010. Disponível a partir de: < http://www.statsoft.com >. Acesso em jun. 2016

THOMPSON, S. K; SEBER, G. A. F. Adaptive Sampling. New York: John Wiley e Sons, 1996.

Recebido em 10/07/2016

Aceito para publicação em 30/03/2017

Sci. For., Piracicaba, v. 45, n. 115, p. 445-453, set. 2017

DOI: dx.doi.org/10.18671/scifor.v45n115.03 
